\title{
Determination of Norfloxacin Using a Tetraoxocalix[2]arene[2]- triazine Covalently Functionalized Multi-walled Carbon Nanotubes Modified Electrode
}

\author{
Jinjin DANG, ${ }^{*}$ Hong CUI, ${ }^{* \dagger}$ Xiangjun LI, ${ }^{* *}$ and Jialing Zhang ${ }^{* \dagger}$ \\ *School of Public Health, Shanxi Medical University, 56 Xinjian South Road, Taiyuan 030001, China \\ **School of Chemical Sciences, University of Chinese Academy of Sciences, 19A Yuquan Road, Beijing 100049, \\ China
}

\begin{abstract}
This paper presents a sensitive voltametric procedure for the determination of norfloxacin (NF) by a tetraoxocalix[2]arene[2]triazine (TOCT) covalently functionalized multi-walled carbon nanotubes (MWCNTs) modified electrode. The electrochemical sensing of NF was investigated by cyclic voltammetry (CV) and differential pulse voltammetry (DPV). Through a combination of the excellent selective recognition of TOCT and the outstanding electronic properties of MWCNTs, this electrochemical sensor shows excellent sensitivity and high selectivity for an electrochemical detection of NF. The stripping response is highly linear $(R=0.996)$ over the NF concentration range of $0.5-8.0 \mu \mathrm{M}$ with the LOD of $0.1 \mu \mathrm{M}$. The fabricated sensors were successfully applied for quantitative detection of NF in pharmaceutical formulations and human urine samples. A high anti-interference ability to common interferences and satisfactory results were obtained. This is expected to play a huge potential in the real-time monitoring of NF in clinical applications.
\end{abstract}

Keywords Norfloxacin, electrochemical sensor, tetraoxocalix[2]arene[2]triazine, multi-walled carbon nanotubes

(Received April 11, 2019; Accepted May 4, 2019; Advance Publication Released Online by J-STAGE May 10, 2019)

\section{Introduction}

Norfloxacin (NF) belongs to synthetic fluoroquinolone antibiotics, which show high activity against gram-positive and gram-negative infections by inhibiting DNA synthesis and replication. ${ }^{1,2} \mathrm{NF}$ has been widely used to treat respiratory and urinary tract infections, eye and skin infections, gonococcal urethritis, and infectious diarrhea. ${ }^{3}$ However, the abuse of NF may cause some side effects such as allergic reactions and antibiotic resistance, which may cause harm to microorganism and endanger human health. ${ }^{4,5}$ Therefore, studies of NF have attracted great attention.

Many analytical methods have been used in the determination of NF, including spectrophotometry, ${ }^{6}$ fluorometry, ${ }^{7}$ HPLC, ${ }^{8}$ LC/MS, ${ }^{9}$ capillary electrophoresis ${ }^{10,11}$ and electrochemical analysis. ${ }^{12,13}$ Among these methods, the electrochemical method with the advantage of simplicity, a fast response, high sensitivity and low cost has attracted more and more attentions. ${ }^{14-16}$ Therefore, the electrochemical method is promising for NF analysis in complex matrices, especially for the biological samples. However, the detection of NF based on electrochemical methods is still very rare, and some novel electrochemical techniques are still needed to be developed. Electrochemical sensors including a carbon nanotubes (CNTs) modified electrode, ${ }^{17}$ enzymatic rotating biosensor, ${ }^{18}$ electrochemical

$\dagger$ To whom correspondence should be addressed. E-mail: cuihong19872007@126.com(H.C.); zhangjialing1961@ 163.com (J. Z.) immunosensor, ${ }^{15}$ a molecularly imprinted sensor ${ }^{19}$ and nanocomposites modified electrode have been introduced to improve the sensitivity and selectivity of NF. ${ }^{12,16}$ In addition, cyclodextrins as one kind of selective molecular recognition elements have also been successfully used as modifiers for chemically modified electrodes to detect NF. ${ }^{20}$ Compared to cyclodextrin, calix $[n]$ arene generally composed of alternating phenols and methylene groups is another major class of host molecules and it has a hydrophobic and electron-rich cavity, which is well suited for complex formation. In addition, a variety of calix $[n]$ arene derivatives have emerged with excellent performance in separation and sensing applications due to their unique selective recognition ability. ${ }^{21-24}$ However, when used alone in electrochemical sensing, calix $[n]$ arenes have not sufficient conductivity to produce sensitive electroanalysis signals and poor reproducibility was obtained. ${ }^{25-27}$ Most of the calix $[n]$ arenes modified electrodes were based on a polymer membrane ion selective electrode and the recognition targets were mostly metal ions. ${ }^{28}$ Therefore, a new calix $[n]$ arenes modified electrode with high conductivity and good reproducibility needs to be designed.

Electrochemical methods based on nanomaterial modified electrodes, due to their high electrical and optical properties, have attracted attention concerning measurements of biomolecules and drugs over the past decade..$^{29-32}$ CNTs have become preferred in most cases because of their high conductivity and chemical properties, ${ }^{1}$ thus, CNTs can greatly improve the sensitivity of the electrochemical measurements and minimize the detection limit. However, due to the strong vander Waals force between CNTs, the dispersion is very poor 


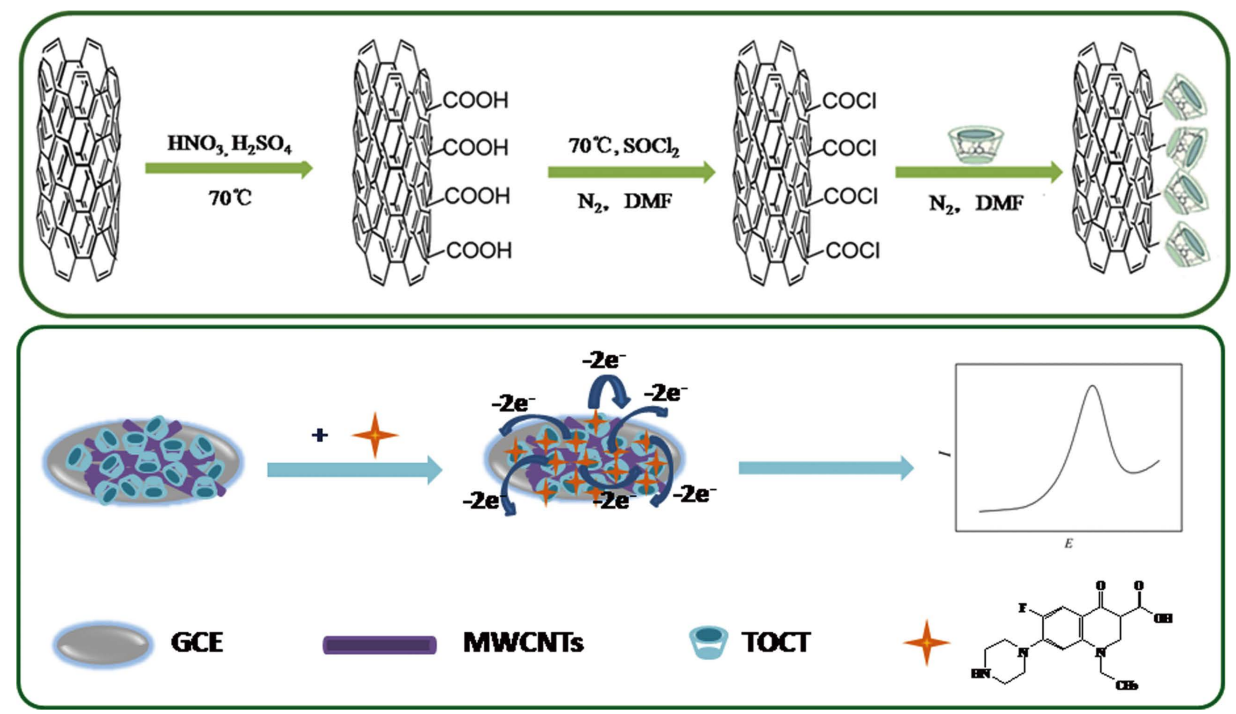

Fig. 1 Schematic diagram of the synthetic procedure for MWCNTs-TOCT, the fabrication process and electroanalysis mechanism of MWCNTs-TOCT/GCE.

in most solvents, and its huge surface area and graphene sheet structure determine that its enrichment effect on many analytes having poor selectivity. Thus it will be a tendency that calixarenes and CNTs coexist and complement each other. To the best of our knowledge, very few efforts have been made concerning the construct of nanocomposites based on CNTs and calixarenes. Özbek et al. modified the calix[4]arene molecules with CNTs with electrostatic bonds for humidity sensing applications. ${ }^{33}$ CNTs were modified with calix[8]arene through a long-time ultrasound treatment and used for ion sensing, however, the complex was only physically mixed. ${ }^{34}$ Wang et al. first reported on the chemical combining of calixarene with CNTs to design a calixarene-CNTs based electrochemical sensor. In the method, thiacalixarene, a new type of calixarene, was introduced and excellent selectivity for $\mathrm{Pb}^{2+}$ was shown. ${ }^{35}$

Oxacalixarene, synthesized from substitution of methylene group by oxygen atom, has appeared as a novel macrocyclic host molecule in the field of supramolecular chemistry. ${ }^{36,37}$ Compared with conventional calixarene, the bridging oxygen atom imparts many novel features and properties; a conjugated system composed of oxygen atoms and aromatic rings is formed. In the present work, tetraoxocalix[2]arene[2]triazine (TOCT), an oxacalix[4]arene derivative, was introduced to form composites by bonding to MWCNTs for the first time. It was anticipated that the selectivity of guest molecules can be improved by the benzene rings, triazine rings, bridging oxygen atoms, the conjugated system and the cavity formed by all. Herein, we have combined the outstanding electrical conductivity of CNTs with the expected selectivity of TOCT by chemically bonding TOCT to CNTs to construct a new sensor for the determination of NF. The composite material was first characterized by FT-IR spectra, X-ray diffraction (XRD) and X-ray photoelectron spectroscopy (XPS), and then highly sensitive electrochemical sensors for NF were successfully fabricated by the composite material, abbreviated as MWCNTsTOCT/GCE. The constructed electrochemical sensor of NF was evaluated using cyclic voltammetry $(\mathrm{CV})$ and differential pulse voltammetry (DPV). The fabrication and electroanalysis mechanism of MWCNTs-TOCT/GCE for the detection of NF are described in Fig. 1. Compared with the MWCNTs/GCE, an enhanced sensitivity was obtained on the MWCNTs-TOCT/
GCE, with a relatively low detection limit for NF $(0.1 \mu \mathrm{M})$, which is the result of the synergetic effect of MWCNTs and TOCT. The developed method was also successfully applied to detect NF in pharmaceutical formulations and human urine samples with satisfactory results without any interference.

\section{Experimental}

\section{Reagents and chemicals}

MWCNTs with a diameter of $10-30 \mathrm{~nm}$ and a length of several micrometers, was purchased from Shenzhen Nanotech Port Co., Ltd. (Shenzhen, China). TOCT was donated by the Dexian Wang research group. ${ }^{37}$ Norfloxacin standards were obtained from Beijing institute for drug control (Beijing, China). All other chemicals were of analytical grade. A $1.0 \mathrm{mM}$ stock standard solution of $\mathrm{NF}$ was prepared in $0.01 \mathrm{M} \mathrm{HCl}$ solution. Working standard solutions were made from the stock standard solution, diluted with buffer solutions as required. A $0.1 \mathrm{M}$ HAc-NaAc buffer (pH 5.0) was used as the supporting electrolyte in all electrochemical experiments. The HAc-NaAc buffer and the stock solution of NF were kept at $4^{\circ} \mathrm{C}$ when not in use. Ultrapure water $(18.25 \mathrm{M} \Omega \mathrm{cm})$ was used for preparation of all solutions and washing.

\section{Apparatus}

All voltammetry measurements and electrochemical impedance spectroscopy (EIS) were performed using a CHI660A electrochemistry workstation ( $\mathrm{CH}$ Instrument, Shanghai) with a conventional three-electrode system. MWCNTs and MWCNTsTOCT modified glassy carbon electrodes (GCE, $3 \mathrm{~mm}$ diameter) were served as the working electrode, while a platinum wire and a saturated calomel electrode (SCE) were used as an auxiliary electrode and a reference electrode, respectively. All of the electrochemical measurements proceeded at room temperature.

IR spectra were recorded on a Bruker Vertex 70 FT-IR spectrometer. X-ray diffraction (XRD) were performed on a MSAL-XD2 using $\mathrm{Cu} K \alpha(1.5406 \AA)$ radiation within a $2 \theta$ range from 10 to $80^{\circ}$ at a step width of $0.01^{\circ}$. The X-ray photoelectron spectroscopies (XPS) were performed using a PHI Quantera $\mathrm{SXM}^{\mathrm{TM}}$ Scanning X-ray Microprobe ${ }^{\mathrm{TM}}$. UV spectra 
A
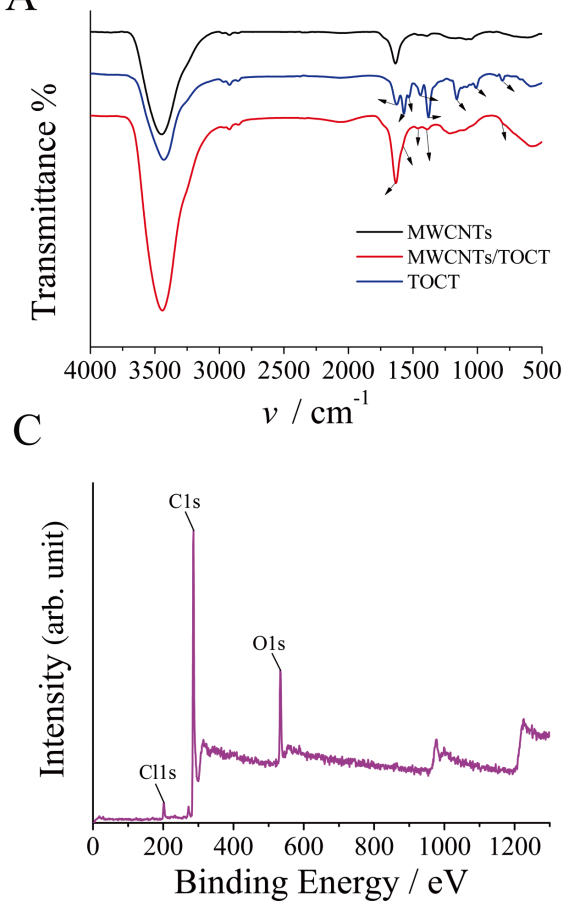

$\mathrm{B}$

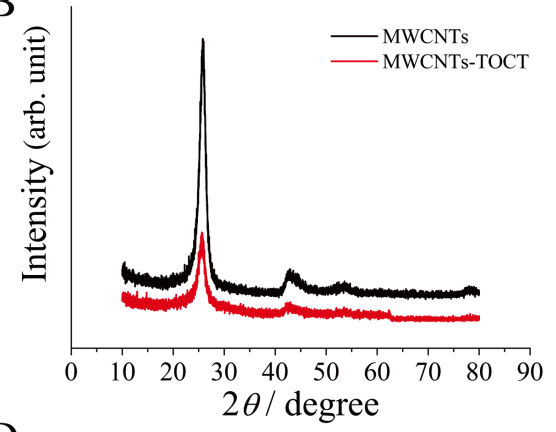

$\mathrm{D}$

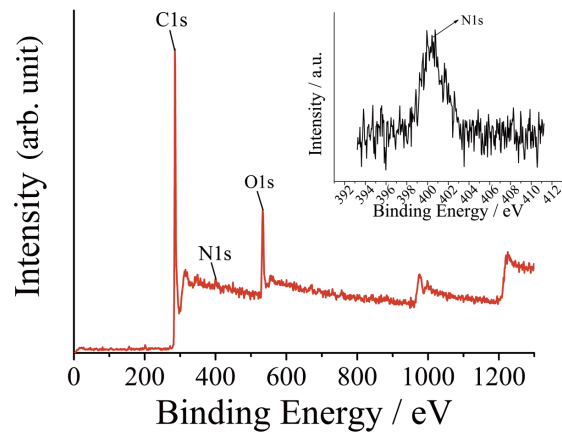

Fig. 2 (A) FT-IR spectra of MWCNTs, TOCT and MWCNTs-TOCT. (B) XRD patterns of MWCNTs and MWCNTs-TOCT. (C) XPS spectra of MWCNTs-COCl. (D) XPS spectra of MWCNTs-TOCT.

were measured with a UV-2550 spectrophotometer (Shimadzu, Japan). All of the pH measurements were performed with a PB-10 pH meter (Sartorius, Germany).

\section{Synthesis of MWCNTs-TOCT}

First, $0.2 \mathrm{~g}$ MWCNTs was added to a mixture of $30 \mathrm{~mL}$ of concentrated $\mathrm{H}_{2} \mathrm{SO}_{4}$ and $10 \mathrm{~mL}$ of concentrated $\mathrm{HNO}_{3}$, and then mixed by ultrasound. The suspension was fully stirred at $70^{\circ} \mathrm{C}$ for $2 \mathrm{~h}$, afterwards diluted with plenty of water and centrifuged at $6000 \mathrm{rpm}$ after cooled to room temperature. The precipitate was washed with ultrapure water repeatedly by sand core funnel filtration until all of the acids were removed. The carboxylated product MWCNTs-COOH was obtained after drying. Then, $0.1 \mathrm{~g}$ MWCNTs-COOH was added to $20 \mathrm{~mL}$ of sulfoxide chloride $\left(\mathrm{SOCl}_{2}\right)$, and $1 \mathrm{~mL}$ of anhydrous $N, N$-dimethylformamide (DMF) was added as both the solvent and catalyst. The mixture was fully stirred overnight at $70^{\circ} \mathrm{C}$ under an argon atmosphere. The unreacted $\mathrm{SOCl}_{2}$ was then removed by distillation and filtration with anhydrous DMF to give MWCNTs-COCl. Subsequently, $0.01 \mathrm{~g}$ TOCT was dissolved in $5 \mathrm{~mL}$ of DMF, and $40 \mu \mathrm{L}$ of anhydrous trimethylamine (TEA) was added as the catalyst, and the mixture was stirred at $70^{\circ} \mathrm{C}$ for $24 \mathrm{~h}$ under argon atmosphere, then a well dispersed black suspension was produced. The suspension was filtered, washed repeatedly with DMF to remove the unreacted TOCT, and then washed with ultrapure water. The final product MWCNTsTOCT was obtained after drying. The synthesis scheme is presented in Fig. 1.

Preparation of the electrochemical sensor for NF measurement Prior to modification, the GCE surface was carefully polished with fine $\mathrm{SiC}$ paper and $0.05 \mu \mathrm{m}$ alumina slurry, then sonicated for about several minutes in ethanol and ultrapure water sequentially, and finally dried by $\mathrm{N}_{2}$ blowing. The immobilizing solution was prepared by dissolving $0.5 \mathrm{mg}$ of MWCNTs-
TOCT into $1 \mathrm{~mL}$ ultrapure water and sonicating for $10 \mathrm{~min}$. The samples became extremely homogeneous black suspensions compared with the MWCNTs solution. An $8 \mu \mathrm{L}$ MWCNTsTOCT solution was dropped directly onto freshly prepared GCE, and allowed to dry under on the infrared lamp.

The new modified electrode was activated in the acetate buffer by successive $\mathrm{CV}$ scanning at $+0.3-+1.6 \mathrm{~V}$ until a stable $\mathrm{CV}$ was obtained, and then transferred into another acetate buffer solution containing a certain concentration of NF. In the DPV analysis, the modified GCE was immersed into the HAc-NaAc buffer containing a certain concentration of $\mathrm{NF}$ to allow preconcentration at an open-circuit potential with $3 \mathrm{~min}$ of stirring. After holding the voltage at $-1.0 \mathrm{~V}$ for $90 \mathrm{~s}$, the stripping process was carried out from +0.6 to $+1.4 \mathrm{~V}(v s$. SCE) at the following parameters: increment, $0.004 \mathrm{~V}$; amplitude, $0.05 \mathrm{~V}$; sampling width, $0.0167 \mathrm{~V}$; pulse period, $0.2 \mathrm{~s}$; pulse width, $0.05 \mathrm{~s}$. For electrode regeneration, the modified electrode was transferred to a blank HAc-NaAc solution and series of scans were continued until the target peak disappeared, and then rinsed with ultrapure water. The above process was also performed for the GCE and MWCNTs/GCE.

\section{Results and Discussion}

\section{Characterization of MWCNTs-TOCT}

The materials of TOCT and MWCNTs-TOCT were characterized using FT-IR spectroscopy. As can be seen from Fig. 2A, the spectrum of TOCT has absorption bands at 1630, 1573 and $1529 \mathrm{~cm}^{-1}$ for the vibration absorption of the benzene and triazine ring skeleton, $807 \mathrm{~cm}^{-1}$ for the characteristic absorption peak of the triazine, $1380,1444 \mathrm{~cm}^{-1}$ for deformation vibration of phenmethyl, and 1008, $1158 \mathrm{~cm}^{-1}$ for Ar-O-Ar. After being bonded to the MWCNTs, a strong absorption band at 1630 and $1573 \mathrm{~cm}^{-1}$ juts out from the prior peak for the 
A

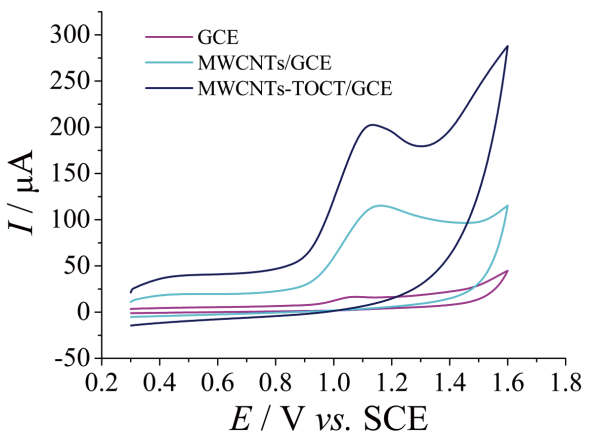

B

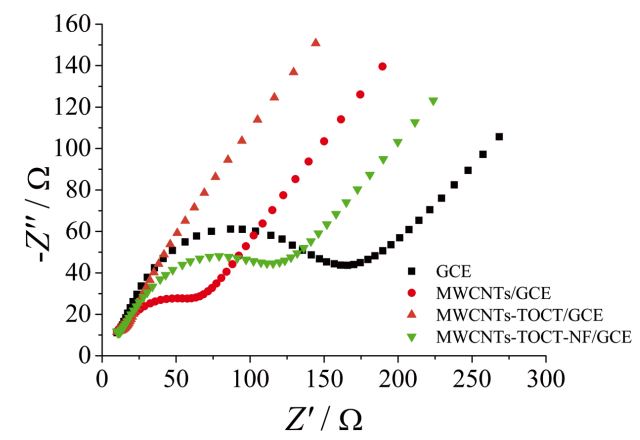

Fig. 3 (A) CVs of $100 \mu \mathrm{M}$ NF in $0.1 \mathrm{M}$ HAc-NaAc (pH 5.0) at bare GCE, MWCNTs-GCE and MWCNTs-TOCT/GCE. (B) Nyquist plots of $5.0 \mathrm{mM}\left[\mathrm{Fe}_{3}(\mathrm{CN})_{6}\right]^{3-14-}$ in $0.1 \mathrm{M} \mathrm{KCl}$ at bare GCE, MWCNTs-GCE and MWCNTs-TOCT/GCE, and the initial potential was set at the same value as open circuit potential.

vibration absorption of the benzene and triazine ring skeleton are observed, $1451,1379 \mathrm{~cm}^{-1}$ corresponding to phenmethyl, and $1044,1201 \mathrm{~cm}^{-1}$ are the equals of Ar-O-Ar. All these absorption bands in MWCNTs-TOCT spectrum confirm the covalent linkage of TOCT to the MWCNTs.

Figure 2B shows the XRD grams of MWCNTs and MWCNTsTOCT. A tall narrow peak at $25.8^{\circ}$ is observed in MWCNTs while this peak becomes much shorter and broader in MWCNTsTOCT. This phenomenon indicates that the crystallinity of the MWCNTs has decreased greatly after the functionalization of TOCT. These XRD curves suggesting that the TOCT has been successfully covalently bound to the MWCNTs.

XPS spectra of MWCNTs and MWCNTs-TOCT are compared in Figs. 2C and 2D. When $\mathrm{SOCl}_{2}$ reacted with carboxylic groups on the MWCNTs, the $-\mathrm{OH}$ group was replaced by chloride, which would lead to a lower oxygen content, and an increasing chlorine content on the surface of MWCNTs. The XPS spectra of MWCNTs-COCl exhibit three peaks for C, O and $\mathrm{Cl}$. After MWCNTs-COCl reacted with TOCT there was no $\mathrm{Cl}$ peak present and an $\mathrm{N}$ peak appeared at same time, which supports the conclusion that the TOCT successfully reacted with MWCNTs-COCl to form MWCNTs-TOCT.

\section{UV spectra characteristics of NF and TOCT}

A weak absorption of TOCT in the $248-320 \mathrm{~nm}$ regions is observed from Fig. S1A (Supporting Information) on account of a small concentration of $1.0 \times 10^{-6} \mathrm{M}$. The UV absorption spectra of NF in NaAc-HAc buffer solution (pH 5.0) at various concentrations of TOCT are also shown in Fig. S1B (Supporting Information). As can be seen, the absorption peak of NF is at $274 \mathrm{~nm}$. However, as the concentration of TOCT added to the NF solution gradually increases, the absorption intensity of NF increases obviously. Meanwhile, the absorption peak of NF gradually moves from 274 to $285 \mathrm{~nm}$, and the absorption peak of TOCT also shows a significant red shift. These phenomena fully prove the interaction between TOCT and NF.

\section{Electrochemical behavior of $\mathrm{NF}$ on different electrodes}

The CVs of $100 \mu \mathrm{M}$ NF at a bare GCE, MWCNTs/GCE and MWCNTs-TOCT/GCE in 0.1 M HAc-NaAc buffer ( $\mathrm{pH}$ 5.0) are shown in Fig. 3A. It can be clearly seen that there was no significant peak current for the oxidation of NF at the bare GCE; while at MWCNTs/GCE, a considerable increase of the oxidation peak for NF was observed at around $+1.1 \mathrm{~V}$ due to the good conductivity and increased surface area of MWCNTs.
Compared to the MWCNTs/GCE, the MWCNTs-TOCT/GCE exhibits a more outstanding increase in the oxidation peak current, indicating that the TOCT provided more active sites for $\mathrm{NF}$, which is consistent with the results of UV spectra. Thus we conclude that the remarkable signal-enhancing effect of the MWCNTs-TOCT derived from the synergetic effect between MWCNTs and TOCT. The CV curves also show that no reduction peak is showed in the reverse scan, suggesting that the electrochemical reaction of NF is a totally irreversible process.

Electrochemical impedance spectroscopy (EIS) is an effective method for exploring the surface characteristics of modified electrodes. Figure 3B compares the Nyquist plots for bare GCE, MWCNTs/GCE and MWCNTs-TOCT/GCE in $5.0 \mathrm{mM}$ $\left[\mathrm{Fe}(\mathrm{CN})_{6}\right]^{3-14-}$ containing $0.1 \mathrm{M} \mathrm{KCl}$ recorded at the open circuit potential. In EIS, the semicircle segment appeared at higher frequencies corresponds to the electron transfer limited process with the diameter equal to charge transfer resistance $\left(R_{\mathrm{ct}}\right)$, and the linear segment observed at lower frequencies attributes to the diffusion limited electron transfer process. As can be seen from Fig. 3B, a semicircle is shown at the bare GCE, while the MWCNTs/GCE exhibits a decreased semicircle indicating that MWCNTs accelerated the electron transfer as an excellent conductive material. However, when TOCT is bonded with MWCNTs, there is almost no obvious semicircle at the time that the $R_{\mathrm{ct}}$ value is close to zero. TOCT surprised provided an easier electron conducting pathway which may be interpreted as the interactions between the aromatic rings of TOCT and MWCNTs have changed the electronic transfer of MWCNTs. An increased semicircle was present at the MWCNTs-TOCT/ GCE after the accumulation of NF. In addition, the higher the concentration of NF, the bigger the impedance, this is not shown in the graph.

\section{Effect of the pH of buffer solution}

The peak currents and peak shapes are closely associated with the $\mathrm{pH}$ of the buffer solution. Therefore, the selection of the $\mathrm{pH}$ of buffer solution is very important for the detection sensitivity. The HAc-NaAc buffer our predecessors used was selected in this study. The buffering capacity of a concentration of $0.1 \mathrm{M}$ was large enough, because maximum peak height was obtained at this concentration. Various pHs $(4.0-6.0)$ of $0.1 \mathrm{M} \mathrm{HAc}-$ $\mathrm{NaAc}$ were studied at MWCNTs-TOCT/GCE (Fig. S2A, Supporting Information). It is clearly seen that both the peak potential $\left(E_{\mathrm{p}}\right)$ and peak current $\left(I_{\mathrm{p}}\right)$ vary with $\mathrm{pH}$ values. The peak current is highest at $\mathrm{pH} 5.0$ and the peak potential shifts 
A

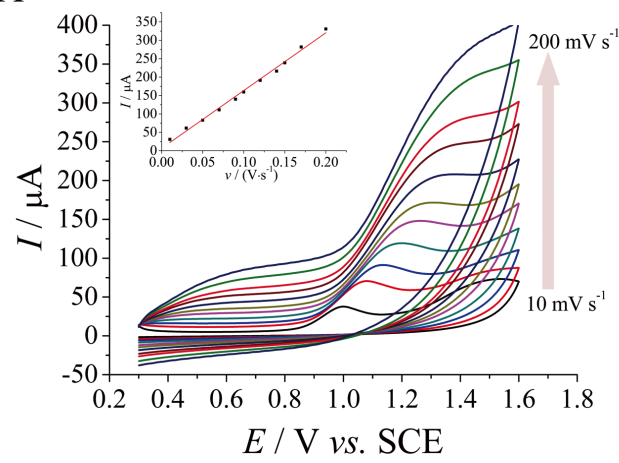

B

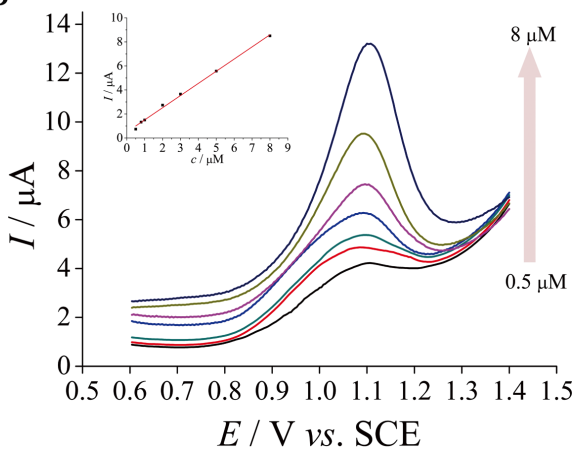

Fig. 4 (A) CVs in the presence of $100 \mu \mathrm{M} \mathrm{NF}$ in $0.1 \mathrm{M} \mathrm{HAc-NaAc}(\mathrm{pH}$ 5.0) at MWCNTs-TOCT/ GCE at different scan rates of $10-200 \mathrm{mV} \mathrm{s}^{-1}$. Inset: Linear relation of I versus v. (B) DPVs of NF in $0.1 \mathrm{M} \mathrm{HAc-NaAc}(\mathrm{pH} 5.0)$ at MWCNTs-TOCT/GCE at different concentration. Inset: the linear relationship of $I$ versus the concentration of $\mathrm{NF}$ of $0.5-8.0 \mu \mathrm{M}$.

Table 1 Comparison of the fabricated sensor with reported sensors for NF determination

\begin{tabular}{lcllc}
\hline \multicolumn{1}{c}{ Modified electrode } & LOD/ $\mu \mathrm{M}$ & \multicolumn{1}{c}{ Linear range/ $\mu \mathrm{M}$} & \multicolumn{1}{c}{ Verified applications } & Reference \\
\hline CuO/MWCNTs/GCE & 0.321 & $1.0-47.7$ & \multicolumn{1}{c}{ None } & 12 \\
P- $\beta$-CD-1-arg/CPE & 0.04 & $0.1-4002$ & Pharmaceutical and serum & 20 \\
MWCNT-CPE/pRGO-ANSAg/Au & 0.016 & $0.03-1.0$ and $1.0-50.0$ & Pharmaceutical and plasma & 38 \\
MWCNTs/Nafion-GCE & 0.05 & $0.1-100$ & Pharmaceutical and urine & 17 \\
MWCNTs-TOCT/GCE & 0.1 & $0.5-8.0$ & Pharmaceutical and urine & This work \\
\hline
\end{tabular}

towards more negative direction as the buffer $\mathrm{pH}$ increases (Fig. S2B, Supporting Information), which means the anodic reaction is more likely to occur under weak acidic conditions. The relationship between $E_{\mathrm{p}}$ and $\mathrm{pH}$ could be expressed as an equation: $E_{\mathrm{p}}=1.53-0.06 \mathrm{pH}$ with excellent linearity of $R^{2}=0.994$. The slope of $-0.06 \mathrm{~V} / \mathrm{pH}$ is in close proximity to the theoretical value, indicating that the number of electrons and protons involved in the charge transfer was equal, being consistent with previously reported results. ${ }^{1,2,17,38}$

\section{Accumulation conditions studies}

Considering the adsorption degree of NF on the electrode surface, NF was firstly accumulated by complexation with TOCT on the surface of the MWCNTs-TOCT/GCE from the solution at open-circuit case, and then continuously accumulated by applying a constant voltage and time. The effect of accumulation time at open circuit condition was investigated between 0 and 6 min. From Fig. S3A (Supporting Information) the stripping signals increase rapidly initially with the accumulation time and reached a plateau at $2 \mathrm{~min}$. This suggests that $2 \mathrm{~min}$ is adequate for the complexation reaction between TOCT and NF. Therefore, $2 \mathrm{~min}$ was choosed as the accumulation time for all follow experiments. The dependence of peak currents on the electro-deposition time was also studied at a concentration of $100 \mu \mathrm{M} \mathrm{NF}$, and $90 \mathrm{~s}$ was selected for further experiments (Fig. S3B, Supporting Information). The electro-deposition potential is also a major factor which affects the response sensitivity. It was evaluated by a concentration of $100 \mu \mathrm{M}$ NF with an accumulation time of $90 \mathrm{~s}$ (Fig. S3C, Supporting Information). The graph shows that the peak currents increases while the peak potential ranges from 0.0 to $-1.2 \mathrm{~V}$; the peak potentials shift to the negative direction from 0.0 to $-1.0 \mathrm{~V}$ then starts to the positive direction. Thus, the electro-deposition potential of $-1.0 \mathrm{~V}$ is the best compromise.

\section{Influence of scan rate}

To reveal the reaction mechanism, the relationship between the peak current and the scan rate was probed, and is shown in Fig. 4A. The peak currents is proportional to the scan rates ranging from 10 to $200 \mathrm{mV} \mathrm{s}^{-1}$, with a calibration equation of $I=6.455+1569.921 v\left(R^{2}=0.993\right)$, indicating that an adsorption control process occurred on the electrode. As the scan rate increases, the peak potential shifts slightly toward a more positive direction, which is a typical performance for the irreversibility of the electrochemical oxidation of NF.

\section{Calibration curve of $N F$}

The analytical performance of the MWCNTs-TOCT/GCE was examined by DPV in different concentrations of NF solutions under the optimized conditions. As shown in Fig. 4B, a good liner relationship between the currents and the concentration of $\mathrm{NF}$ was found in the range from 0.5 to $8.0 \mu \mathrm{M}$, and the regression equation is expressed as $I=0.50+1.01 \mathrm{c}\left(R^{2}=\right.$ 0.996). The detection limit of NF at the MWCNTs-TOCT/GCE was $0.1 \mu \mathrm{M}(S / N=3)$ calculated by using the formula $3 \sigma / b$, where $\sigma$ represents the standard deviation of the signal of the blank solution and $b$ symbolizes the slope of the curve. A comparison of this method with other reported methods for NF detection was summarized in Table 1. The results show that the preparation of the electrode modified material was really quite simple. Therefore, the electrochemical sensor will become a valuable tool for NF detection, and deserves extensive application.

\section{Interference, reproducibility and stability}

The aim of the interference study is to explore the effects of possible coexistent species in the determination of NF, so we selected some inorganic ions and biological molecules that might coexist with NF for simultaneous determination. 
Table 2 Determination of NF in pharmaceutical formulations and human urine $(n=3)$

\begin{tabular}{ccccc}
\hline Sample & Added $/ \mu \mathrm{M}$ & Found/ $\mu \mathrm{M}$ & Recovery, $\%$ & RSD, \% \\
\hline \multirow{2}{*}{ Tablets } & 1.00 & 1.00 & 100.00 & 8.63 \\
& 5.00 & 4.99 & 99.80 & 8.39 \\
\multirow{2}{*}{ Urine } & 8.00 & 7.52 & 94.00 & 4.34 \\
& 1.00 & 1.00 & 100.00 & 3.64 \\
& 5.00 & 5.47 & 109.50 & 4.72 \\
& 8.00 & 7.96 & 99.50 & 2.86 \\
\hline
\end{tabular}

The results indicated that 100 -fold concentration of $\mathrm{Na}^{+}, \mathrm{K}^{+}$, $\mathrm{Cu}^{2+}, \mathrm{Ca}^{2+}, \mathrm{Zn}^{2+}, \mathrm{Fe}^{3+}, \mathrm{Al}^{3+}, \mathrm{Cl}^{-}, \mathrm{NO}_{3}{ }^{-}, \mathrm{HPO}_{4}{ }^{2-}, \mathrm{SO}_{4}{ }^{2-}, 20$-fold concentration of uric acid, ascorbic acid, adrenaline, dopamine, glucose, tyrosine, and 10-fold concentration of tryptophan had little effect on the current response of $10 \mu \mathrm{M}$ NF. It suggested that the established method has good selectivity for NF.

The reproducibility of the same MWCNTs-TOCT/GCE was examined by five successive determinations of $8 \mu \mathrm{M} \mathrm{NF}$, and RSD of $5.36 \%$ was obtained. To further check the reproducibility of the results, five different MWCNTs-TOCT/GCEs were prepared and used for the measurement of $8 \mu \mathrm{M} \mathrm{NF}$, and an acceptable reproducibility with a RSD of $9.19 \%$ was obtained. The stability of the MWCNTs-TOCT/GCE was also tested. After one measurement, the current was measured over a period of $24 \mathrm{~h}$ during one week. The peak current intensity only decreased $4.4 \%$ after one week, indicating that the MWCNTsTOCT/GCE possessed well stability.

Analysis of NF in human urine and pharmaceutical formulations With the purpose to evaluate the applicability of the developed method, urine samples obtained from healthy male which were diluted directly 10-folds with $0.1 \mathrm{M} \mathrm{HAc-NaAc}$ buffer ( $\mathrm{pH} 5.0$ ) to minimize matrix complexity were used for analytical assay. The standard recovery test is used to calculate the recovery and evaluate the accuracy of the method. Certain concentrations of $\mathrm{NF}$ were spiked into the urine samples to calculate the recovery and evaluate the accuracy of the methods. The results are tabulated in Table 2, and the satisfactory recoveries obtained in the range from 99.5 to $109.5 \%$.

The developed method was also used to determine the content of NF in commercial capsules (Guangdong Bangmin Pharmaceutical Co., Ltd., China). Each capsule containing $100 \mathrm{mg}$ of $\mathrm{NF}$ were pretreated according to the literature. ${ }^{38}$ DPVs were then carried out under the optimum conditions and the NF recovery was found between 94.0 and $100.0 \%$, indicating the feasibility of this method in the determination of NF content in pharmaceutical samples. All of the above data suggest that this method has good application value.

\section{Conclusions}

In summary, a composite material based on MWCNTs-TOCT has been synthesized to construct a sensitive electrochemical sensor, which has been applied for the determination of NF in the pharmaceutical matrix and male urine. The developed MWCNTs-TOCT sensor exhibits the advantages of simple operation, high sensitivity and practicability. The outstanding performance of this electrochemical sensor is due to the excellent selectivity of TOCT and the well-known high conductivity and surface area of MWCNTs. This method also has the characteristics of fast response, good reproducibility and stability, and has high anti-interference a bility to common interferences of pharmaceutical formulations or urine samples, which is expected to play a huge potential in the real time monitoring of NF in clinical applications.

\section{Competing Interests}

We have no competing interests.

\section{Acknowledgements}

This work was supported by the National Natural Science Foundation of China (No. 81803286), Natural Science Foundation of Beijing Municipality (No. 2182083) and Start-up Fundation of Shanxi Medical University (No. 03201516).

\section{Supporting Information}

The additional experimental descriptions and discussions are available in Supporting Information. This material is available free of charge on the Web at http://www.jsac.or.jp/analsci/.

\section{References}

1. B. Agrawal, P. Chandra, R. N. Goyal, and Y. B. Shim, Biosens. Bioelectron., 2013, 47, 307.

2. R. N. Goyal, A. R. S. Rana, and H. Chasta, Bioelectrochemistry, 2012, 83, 46.

3. O. Ballesteros, I. Toro, V. Sanz-Nebot, A. Navalón, J. L. Vílchez, and J. Barbosa, J. Chromatogr. B, 2003, 798, 137.

4. W. H. Sheng, Y. C. Chen, J. T. Wang, S. C. Chang, K. T. Luh, and W. C. Hsieh, Diagn. Microbiol. Infect. Dis., 2002, 43, 141

5. S. Pakpinyo and J. Sasipreeyajan, Vet. Microbiol., 2007, $125,59$.

6. L. V. S. Santos, A. M. Meireles, and L. C. Lange, J. Environ. Manage, 2015, 154, 12.

7. M. Kamruzzaman, A. M. Alam, H. L. Shang, Y. H. Kim, G. M. Kim, and H. O. Sang, J. Lumin., 2012, 132, 3053.

8. C. Mendes, A. Buttchevitz, J. H. Kruger, L. S. Bernardi, P R. Oliveira, and M. A. Silva, Anal. Sci., 2015, 31, 1083.

9. M. Denadai and Q. B. Cass, J. Chromatogr. A, 2015, 1418, 177.

10. A. V. Herreraherrera, L. M. Ravelopérez, J. Hernándezborges, M. M. Afonso, J. A. Palenzuela, and M. Á. Rodríguezdelgado, J. Chromatogr. A, 2011, 1218, 5352.

11. C. C. Liu, X. Feng, H. L. Qian, G. Z. Fang, and S. Wang, Food Anal. Methods, 2015, 8, 596.

12. M. Devaraj, R. K. Deivasigamani, and S. Jeyadevan, Colloids Surf., B, 2013, 102, 554.

13. S. D. Jojoa-sierra, J. Silvaagredo, E. Herreracalderon, and R. A. Torrespalma, Sci. Total Environ., 2017, 575, 1228.

14. R. Zhang, C. L. Sun, Y. J. Lu, and W. Chen, Anal. Chem., 2015, 87, 12262.

15. B. Liu, M. Li, Y. S. Zhao, M. F. Pan, Y. Gu, W. Sheng, G. Z. Fang, and S. Wang, Sensor, 2018, 18, 1946.

16. S. Hamnca, L. Phelane, E. Iwuoha, and P. Baker, Anal. Lett., 2017, 50, 1887.

17. K. J. Huang, X. Liu, W. Z. Xie, and H. X. Yuan, Colloids Surf., B, 2008, 64, 269.

18. A. A. J. Torriero, E. Salinas, J. Rabaa, and J. J. Silber, Biosens. Bioelectron., 2006, 22, 109. 
19. H. D. Silva, J. Pacheco, J. Silva, S. Viswanathan, and C. Delerue-Matos, Sens. Actuators, B, 2015, 219, 301.

20. F. F. Zhang, S. Q. Gu, Y. P. Ding, Z. Zhang, and L. Li, Anal. Chim. Acta, 2013, 770, 53.

21. W. J. Zhao, K. Hu, C. J. Wang, S. Liang, B. L. Niu, L. J. He, K. Lu, B. X. Ye, and S. S. Zhang, J. Chromatogr. A, 2012, 1223, 72.

22. H. Y. Aboul-Enein, H. A. A. Wagdy, and R. M. E. Nashar, Curr. Anal. Chem., 2009, 5, 249.

23. R. Ludwig and N. T. K. Dzung, Sensors, 2002, 2, 397.

24. I. Leray and B. Valeur, Cheminform, 2010, 40, 3525.

25. M. Shamsipur, A. A. Miran Beigi, M. Teymouri, S. Rasoolipour, and Z. Asfari, Anal. Chem., 2009, 81, 6789.

26. V. K. Gupta, A. K. Jain, M. A. Khayat, S. K. Bhargava, and J. R. Raisoni, Electrochim. Acta, 2008, 53, 5409.

27. F. Wang, Y. J. Wu, K. Lu, and B. X. Ye, Electrochim. Acta, 2013, 87, 756.

28. K. M. O'Connor, D. W. M. Arrigan, and G. Svehla, Electroanalysis, 2010, 7, 205.

29. A. A. Abdelwahab, W. C. Koh, H. B. Noh, and Y. B. Shim,
Biosens. Bioelectron., 2010, 26, 1080.

30. Y. Zhu, J. I. Son, and Y. B. Shim, Biosens. Bioelectron., 2011, 26, 1002.

31. D. Ersin, I. Onur, and I. Recai, Anal. Sci., 2018, 34, 771.

32. H. Q. Xia, Y. Kitazumi, O. Shirai, and K. Kano, Anal. Sci., 2017, 33, 839.

33. C. Özbek, E. Culcular, S. Okur, M. Yilmaz, and M. Kurt, Acta Phys. Pol. A, 2013, 123, 461.

34. F. L. Supian, S. A. Bakar, N. A. Azahari, and T. H. Richardson, American Institute of Physics, 2013, 1528, 260.

35. L. Wang, X. Y. Wang, G. Shi, C. Peng, and Y. H. Ding, Anal. Chem., 2012, 84, 10560.

36. Q. Q. Wang, D. X. Wang, H. B. Yang, Z. T. Huang, and M. X. Wang, Chem. Eur. J., 2010, 16, 13053.

37. D. X. Wang, Q. Q. Wang, Y. C. Han, Y. L. Wang, Z. T. Huang, and M. X. Wang, Chemistry, 2010, 16, 7265.

38. Z. P. Liu, M. L. Jin, J. P. Cao, J. Wang, X. Wang, G. F. Zhou, A. V. D. Berg, and L. L. Shui, Sens. Actuators, B, 2018, 257, 1065. 